\title{
Down-regulation of LncRNA TUG1 enhances radiosensitivity in bladder cancer via suppressing HMGB1 expression
}

\author{
Huijuan Jiang ${ }^{*}{ }^{\dagger}$ Xigang $\mathrm{Hu}^{\dagger}$, Hongzhi Zhang and Wenbo Li
}

\begin{abstract}
Background: Long non-coding RNAs (IncRNAs) have been reported to regulate the sensitivity of different cancer cells to chemoradiotherapy. Aberrant expression of IncRNA Taurine-upregulated gene 1 (TUG1) has been found to be involved in the development of bladder cancer, however, its function and underlying mechanism in the radioresistance of bladder cancer remains unclear.

Methods: Quantitative real-time PCR (qRT-PCR) was conducted to measure the expression of TUG1 and HMGB1 mRNA in bladder cancer tissues and cell lines. HMGB1 protein levels were tested by western blot assays. Different doses of X-ray were used for radiation treatment of bladder cancer cells. Colony survival and cell viability were detected by clonogenic assay and CCK-8 Kit, respectively. Cell apoptosis was determined by flow cytometry. A xenograft mouse model was constructed to observe the effect of TUG1 on tumor growth in vivo.

Results: The levels of TUG1 and HMGB1 were remarkably increased in bladder cancer tissues and cell lines. Radiation treatment markedly elevated the expression of TUG1 and HMGB1. TUG1 knockdown inhibited cell proliferation, promoted cell apoptosis and decreased colony survival in SW780 and BIU87 cells under radiation. Moreover, TUG1 depletion suppressed the HMGB1 mRNA and protein levels. Furthermore, overexpression of HMGB1 reversed TUG1 knockdown-induced effect in bladder cancer cells. Radiation treatment dramatically reduced the tumor volume and weight in xenograft model, and this effect was more obvious when combined with TUG1 silencing.
\end{abstract}

Conclusion: LnCRNA TUG1 knockdown enhances radiosensitivity of bladder cancer by suppressing HMGB1 expression. TUG1 acts as a potential regulator of radioresistance of bladder cancer, and it may represent a promising therapeutic target for bladder cancer patients.

Keywords: IncRNA, TUG1, HMGB1, Bladder cancer, Radiosensitivity

\section{Background}

Bladder cancer, the ninth most common cancer worldwide, can be distributed into two clinically different groups, non-muscle invasive bladder cancer (NMIBC) and muscle invasive bladder cancer (MIBC) [1]. Although chemoradiotherapy as an adjuvant treatment of surgery was developed, it failed to universally improve outcomes due to the resistance of bladder cancers to chemoradiotherapy [2]. Therefore, further excavate the potential mechanisms of radioresistance

\footnotetext{
*Correspondence: juanjiangh@163.com

${ }^{\dagger}$ Equal contributors

Department of Radiotherapy, Huaihe Hospital of Henan University, No.1 Baobei Road, Gulou District, Kaifeng 475000, China
}

and improve radiosensitivity in patients with bladder cancer become very urgent.

High mobility group box 1 protein (HMGB1), a chromosome-binding protein, functions as a DNA chaperone and involves in many physiological processes in the cell nucleus, including DNA repair, duplication, transcription, and nucleosome packaging [3, 4]. When translocated to the cytoplasm, HMGB1 could invoke autophagy via interacting with beclin-1 [5]. HMGB1 from the extracellular medium warns surrounding cells and immune system to urgent danger, contributing to inflammation [6]. Overexpression of HMGB1 was observed in several cancers, and played significant roles in regulation of tumor growth, metastasis, and chemotherapy and 
radiotherapy resistance [7-10]. HMGB1 is capable of promoting both chemoresistance and radioresistance in breast cancer cells [11, 12]. However, the function of HMGB1 in bladder cancer carcinogenesis and radioresistance remains poorly understood.

Long non-coding RNAs (lncRNAs) are non-proteincoding transcripts longer than 200 nucleotides, and exert their physiological and pathological functions through their interactions with genomic DNA, miRNAs, mRNAs and proteins [13]. Increasing evidence suggests that lncRNAs are important molecules involved not only in normal development but also in tumorigenesis [14]. Abnormal expression lncRNAs could serve as oncogenes and tumor suppressors, closely associated with tumorigenesis, metastasis, prognosis or diagnosis [15]. Moreover, accumulating documents reveal that lncRNAs are related to radiotherapy resistance of cancers [16-18]. For instance, HOTAIR expression was upregulated in tumor tissues of colorectal cancer (CRC) patients, and HOTAIR knockdown inhibited proliferation, migration and invasiveness while enhanced apoptosis and radiosensitivity of CRC cells [18]. HOTAIR expression was markedly increased in the pancreatic ductal adenocarcinoma (PDAC) cell lines and tissues, and HOTAIR silencing improved the radiosensitivity of PDAC cells via regulating the expression of Wnt inhibitory factor 1 (WIF-1) [16].

Taurine-upregulated gene 1 (TUG1) was firstly reported to be upregulated in exposure to the treatment of taurine in mouse retinal cells [19]. TUG1 has been proved to act as a tumor suppressor or oncogene in various cancers [20-22]. In addition, aberrant expression of TUG1 has been observed in bladder cancer cells. For instance, TUG1 expression was remarkably increased in high-grade MIBC tumor tissues, and TUG1 silencing suppressed proliferation and migration in high-grade MIBC [23]. TUG1 was upregulated in bladder cancer tissues and cell lines, and promoted cancer cell invasion and radioresistance through inducing epithelial-to-mesenchymal transition (EMT) [24]. However, the function and molecular mechanism of TUG1 in bladder cancer radioresistance is still largely undefined.

In the present study, we aimed to investigate the effect of TUG1 on the radiosensitivity of bladder cancer cells and its underlying molecular mechanisms.

\section{Methods}

\section{Patient samples and cell lines}

Thirty-nine pairs of bladder cancer tissues and matched adjacent normal tissues were obtained from Huaihe Hospital of Henan University. The Clinical characteristics of patients with bladder cancer were shown in Table 1. Written consents from all patients and approval of Huaihe Hospital Ethic Review Committees were obtained prior to the use of these clinical materials.
Table 1 Patient demographics and clinical characteristics

\begin{tabular}{ll}
\hline $\begin{array}{l}\text { Demographic or clinical characteristic } \\
\text { Age, years }\end{array}$ & $\begin{array}{l}\text { No. of patients } \\
(N=39)\end{array}$ \\
Median & 67 \\
Range & $56-89$ \\
Sex & \\
Male & 27 \\
Female & 12 \\
Primary site & \\
Bladder & 34 \\
Renal pelvis & 5 \\
Histology & \\
TCC & 32 \\
SCC/adenocarcinoma/neuroendocrine & 7 \\
Stage ${ }^{a}$ & \\
Ocis/Oa/1 & 5 \\
2/3 & 18 \\
4 & 16 \\
\hline Abbreviations: $T C C$ Transitional cell carcinoma, SCC Squamous cell carcinoma \\
aStages are defined as follows: Ocis = carcinoma in situ; 0a = noninvasive \\
papillary carcinoma; 1 = tumor invades subepithelial connective tissue; $2 / 3=$ \\
invasive tumor, muscle/perivesicular tissue; and $4=$ metastatic disease
\end{tabular}

Normal bladder epithelial cell line (HCV-29) and human bladder cancer cell lines (SW780, HT1376, BIU87 and T24) were obtained from the American Type Culture Collection (ATCC) and cultured at $37^{\circ} \mathrm{C}$ in humidified 5\% CO2. All cell lines were cultured in RPMI 1640 (Gibco, Grand Island, NY, USA) supplemented with $10 \%$ fetal bovine serum, $100 \mathrm{U} / \mathrm{mL}$ penicillin, and $100 \mathrm{mg} /$ $\mathrm{mL}$ streptomycin.

\section{Quantitative real-time PCR (qRT-PCR)}

TRIzol reagent (Invitrogen, Carlsbad, CA, USA) was used to extract the total RNA from bladder cancer tissues and cell lines. cDNAs were synthesized by reverse transcription using M-MLV reverse transcriptase (Invitrogen). Oligo (dT18) RT primer was used for the reverse transcription of HMGB1 mRNA and lncRNA TUG1. qRT-PCR analysis was performed using SYBR Premix Ex Taq II (TaKaRa, Dalian, China) with an ABI 7500 Real-Time PCR system (Applied Biosystems, Foster City, CA, USA). $\beta$-actin was used as endogenous controls. The gene specific primers were as follows: LncRNA TUG1 (forward: 5'-CTGAAGAAAGGCAACATC-3'; reverse: 5'-GTAGGCTACTACAGGATTTG-3'); HMGB1 (forward: 5'-ACATCCAAAATCTTGATCAGTTA-3'; reverse: 5'-AGGACAGACTTTCAAAATGTTT-3'); $\beta$-actin (forward: 5'TGAGAGGGAAATCGTGCGTGAC-3'; reverse: 5'-AAGAAGGAAGGCTGGAAAAGAG-3'). 


\section{Western blot analysis}

Protein concentrations in the whole-cell lysates were detected by using a BCA Protein Assay Kit (Pierce, Rockford, IL, USA). Then, protein samples were separated by 10\% SDS-PAGE and transferred to polyvinylidene fluoride (PVDF) (Amersham Pharmacia, Little Chalfont, UK). The immunoblots were conducted by incubation with specific antibodies for HMGB1 (Cat. No: sc-56698) and $\beta$ actin (Cat. No: sc-47778). All antibodies were purchased from Santa Cruz Technology (Santa Cruz, CA, USA). Image Quant software (Molecular Dynamics, Sunnyvale, CA, USA) was used to analyze the protein bands.

\section{Cell transfection}

Three TUG1 siRNAs (si-TUG1\#1, si-TUG1\#2 and siTUG1\#3) and the scramble negative controls were synthesized and purchased from GenePharma (Shanghai, China). The sequences of the three designed TUG1 siRNAs were as follows: si-TUG1 1\#, CAGUCCUGGUGA UUUAGACAGUCUU; si-TUG12\#, CCCAGAAGUUG UAAGUUCACCUUGA; si-TUG1 3\#, CAGCUGUUAC CAUUCAACUUCUUAA. Preliminary experiments were performed in both SW780 and BIU87 cells transfected with $100 \mathrm{nM}$ different si-TUG1 to identify the most efficient siRNA sequences. To amplify HMGB1, SW780 and BIU87 cells were transfected with $5 \mu \mathrm{g}$ pcDNA-HMGB1 (GenePharma). All transfection was performed using
Lipofectamine 2000 reagent (Invitrogen) according to the manufacturer's instructions.

\section{Clonogenic assay}

Transfected SW780 and BIU87 cells were treated with a range of radiation doses $(0,2,4,6$ and $8 \mathrm{~Gy})$. When there was visible colony by naked eye, cells were fixed with methanol and stained with $1 \%$ crystal violet solution (Sigma, St. Louis, MO, USA) for $20 \mathrm{~min}$. Colonies with more than 50 cells were then counted under an inverted microscope. Sigmaplot software (Systat, San Jose, CA, USA) was used to fit the data to a linear-quadratic model.

\section{Flow cytometry}

The Annexin V-FITC Apoptosis Detection Kit (Sigma) was used to detect cell apoptosis in accordance with the manufacturer's instructions. Briefly, cells were harvested and stained in the dark for $15 \mathrm{~min}$ at room temperature using $5 \mu \mathrm{L}$ Annexin V-FITC and $10 \mu \mathrm{L}$ propidium iodide (PI). Then the BD FACS Diva software V6.1.3 (BD Biosciences, San Jose, CA, USA) was used to analyze the flow cytometry data.

\section{Cell viability assays}

SW780 and BIU87 cells transfected with si-TUG1 or siTUG1 and pcDNA-HMGB1 were seeded in a 96-well plate for $24 \mathrm{~h}$, and then cells were exposed to 2 Gy radiation. At $0,24,48,72$ and $96 \mathrm{~h}$ after radiotherapy, cell
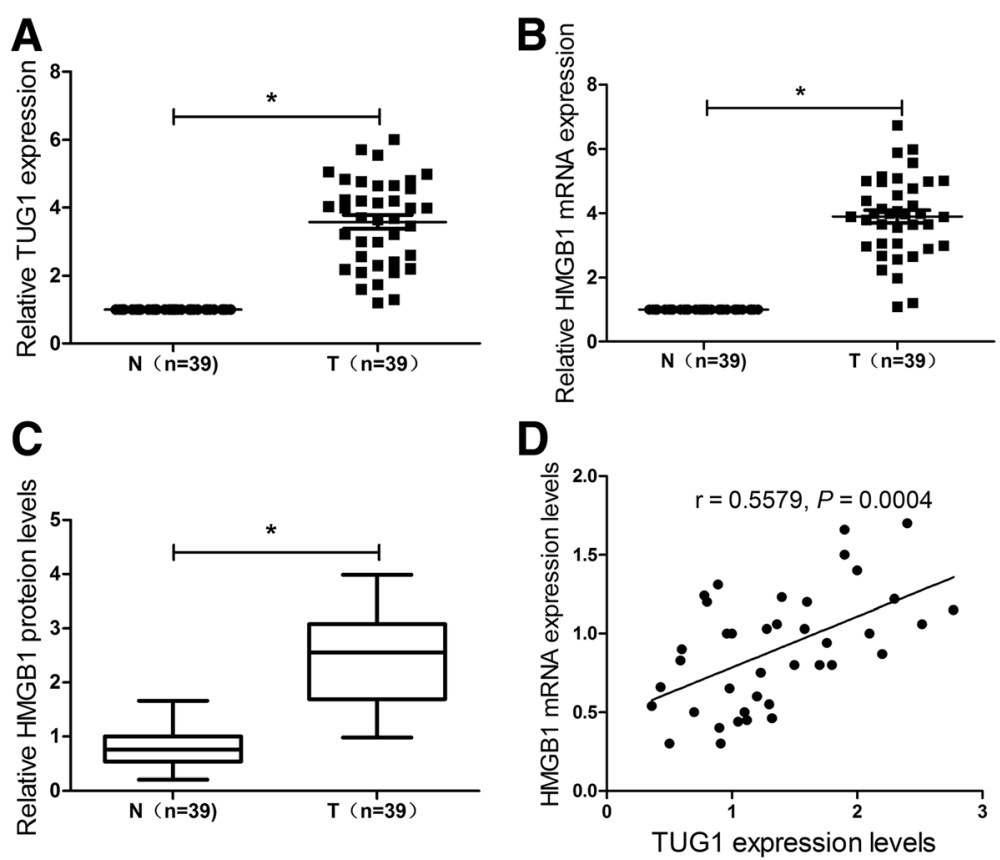

Fig. 1 Expression of TUG1 and HMGB1 in bladder cancer tissues. $\mathbf{a}$ and $\mathbf{b}$ The expression of TUG1 and HMGB1 mRNA was measured by qRT-PCR analysis in bladder cancer tissues $(n=39)$ or adjacent non-cancer tissues $(n=39)$. $\mathbf{c}$ The protein level of HMGB1 was detected by western blot in bladder cancer tissues $(n=39)$ or adjacent non-cancer tissues $(n=39)$. d Correlation analysis of TUG1 and HMGB1 expression in bladder cancer tissues. ${ }^{*} P<0.05$ vs. control 
viability was determined by the Cell Counting Kit- 8 Kit (Dojindo, Kumamoto, Japan) in accordance with the manufacturer's protocol.

\section{Xenograft mouse model}

Total $2 \times 10^{6}$ non-transfected or transfected (si-NC or si-TUG1) SW780 cells were subcutaneously injected into the back of four- to six-week-old male athymic nude mice ( $n=6$ per group). To evaluate tumor radioresistance in vivo, the mice were given radiation (2 Gy) for 5 consecutive days when the tumors reached an average volume of approximately $100 \mathrm{~mm}^{3}$. Tumor volume was measured with slide calipers every three days after the first radiotherapy according to the formula: volume $=1 / 2 \times$ length $\times$ width $^{2}$. Three weeks later, mice were sacrificed to remove and weigh tumors. All animal procedures were performed with the approval of the Local Medical Experimental Animal Care Commission.

\section{Statistical analysis}

Statistical analysis was performed using SPSS 19.0 software (SPSS, Chicago, IL, USA). The data were expressed as means $\pm \mathrm{SD}$. The difference between groups was evaluated by the Student's $t$-test or one-way ANOVA. $P<0.05$ indicated a statistical significance.
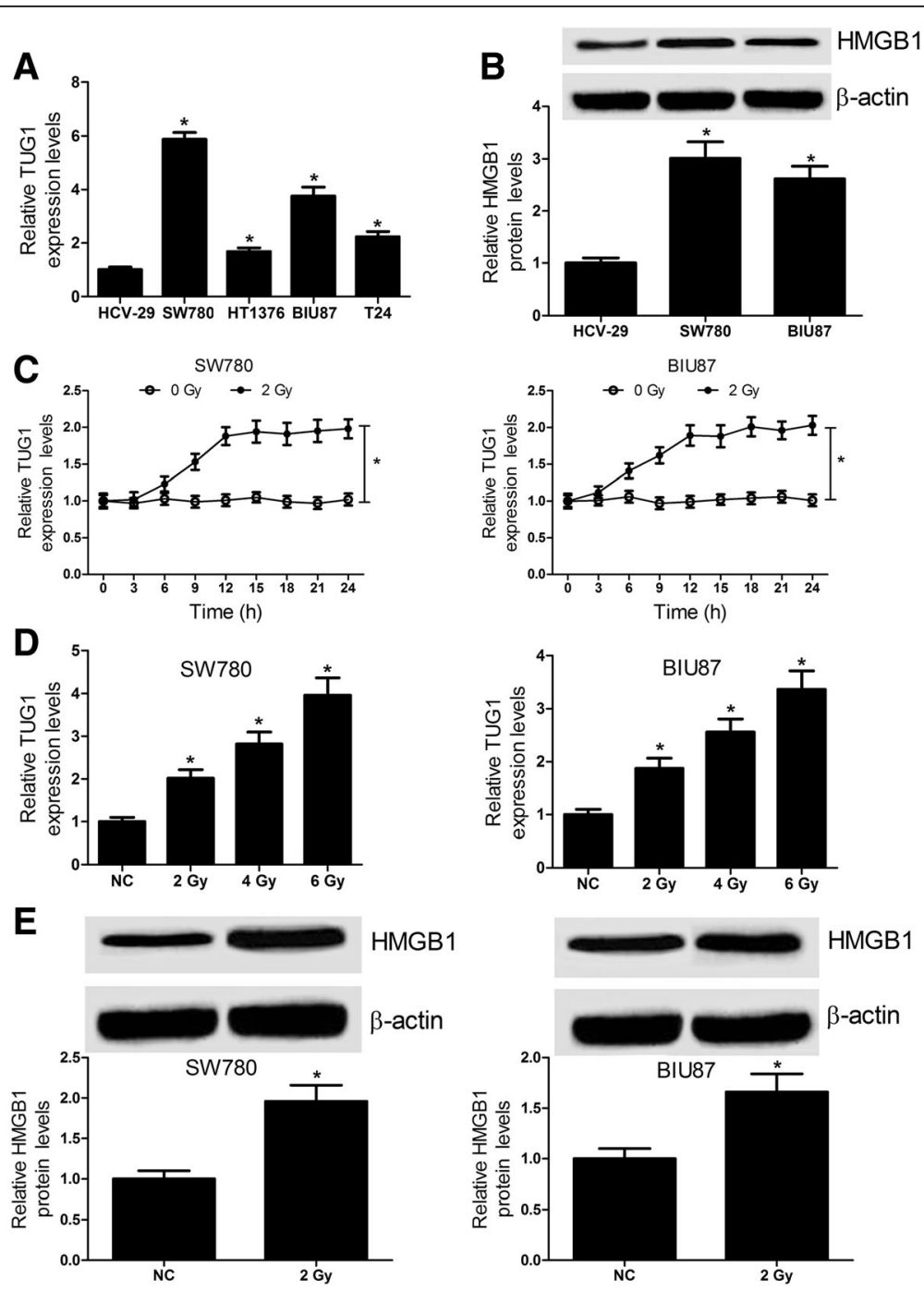

Fig. 2 Radiation promotes the TUG1 and HMGB1 expression in bladder cancer cell lines. a qRT-PCR analysis was conducted to detect the TUG1 expression in bladder cancer cell lines SW780, HT1376, BIU87 and T24 and normal bladder epithelial cell line HCV-29. b The protein level of HMGB1 was determined by western blot in SW780 and BIU87 cells. c TUG1 expression was detected in SW780 and BIU87 cells every 3 h after 0 or 2 Gy of ionizing radiation treatment. d TUG1 expression was measured in SW780 and BIU87 cells after different doses (0, 2, 4, 6 Gy) of ionizing radiation treatment for $24 \mathrm{~h}$. e The HMGB1 protein level was upregulated after SW780 and BIU87 cells treated with 2 Gy of ionizing radiation for 24 h. ${ }^{*} P<0.05$ vs. NC 


\section{Results}

The level of TUG1 is positively correlated with HMGB1 expression in bladder cancer tissues

The expression of TUG1 and HMGB1 mRNA was measured by qRT-PCR and the HMGB1 protein level was determined by western blot in bladder cancer tissues and adjacent non-cancer tissues, respectively. The expression level of TUG1 (Fig. 1a) and HMGB1 mRNA (Fig. 1b) and protein (Fig. 1c) was about twice higher in bladder cancer tissues than that in adjacent normal tissues. Moreover, the level of TUG1 is positively related to HMGB1 expression in bladder cancer tissues (Fig. 1d). These results suggested
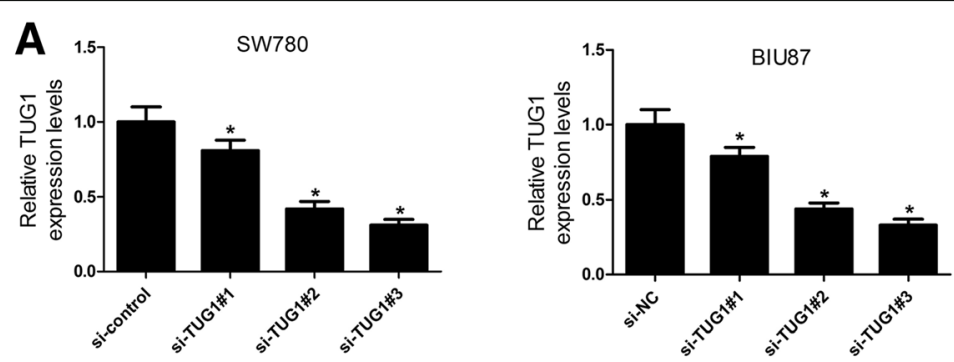

B

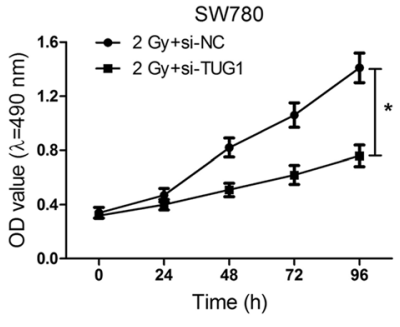

C

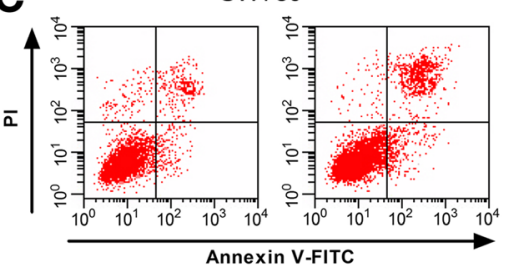

BIU87

D

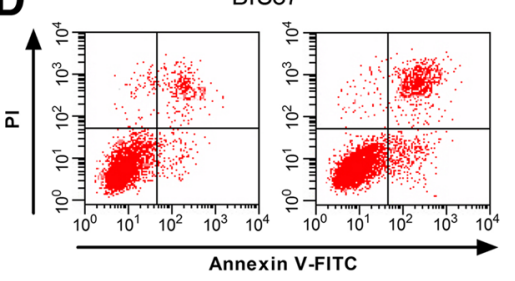

E

SW780 $\rightarrow$ si-NC

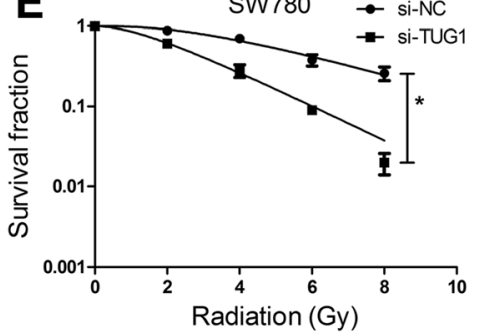

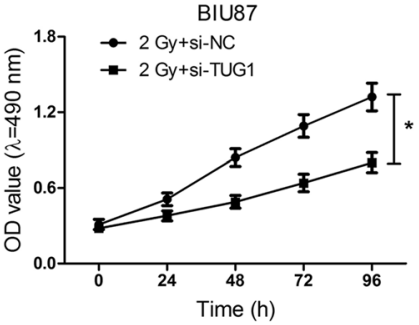
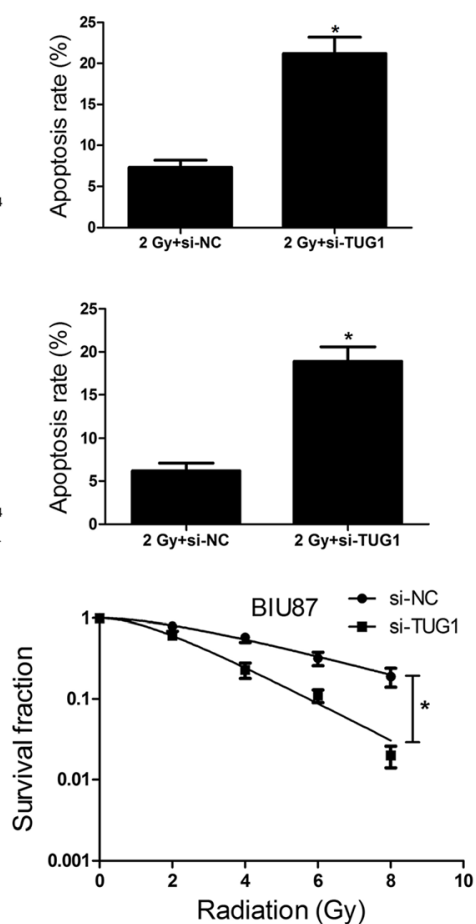

Fig. 3 TUG1 silencing enhances radiosensitivity of bladder cancer cell lines. a qRT-PCR analysis of the relative TUG1 expression in SW780 and BIU87 cells transfected withTUG1-specific si-RNAs or control si-RNA. SW780 and BIU87 cells were transfected with si-NC or si-TUG1 and cultured for $24 \mathrm{~h}$ followed by ionizing radiation. b The cell viability of transfected SW780 and BIU87 cells at 0, 24, 48, 72 and $96 \mathrm{~h}$ afterionizing radiation. c and $\mathbf{d}$ Cell apoptosis was determined in transfected SW780 and BIU87 cells at $24 \mathrm{~h}$ after 2 Gy radiotherapy. e Transfected SW780 and BIU87 cells were subjected to $0,2,4,6$ and 8 Gy of irradiation. After 2 weeks, the colony survival fractions were measured. ${ }^{*} P<0.05$ vs. si-NC 
that upregulation of TUG1 and HMGB1 may be involved in the pathogenesis of bladder cancer.

\section{Radiation treatment increases the TUG1 expression and HMGB1 protein level in bladder cancer cell lines}

To test the expression change of TUG1 and HMGB1 in bladder cancer cell lines, qRT-PCR and western blot were performed, respectively. TUG1 was significantly increased in bladder cancer cell lines (SW780, HT1376, BIU87 and T24) compared with normal bladder epithelial cell line HCV-29 (Fig. 2a), especially in SW780 and BIU87 cells. Similarly, the HMGB1 protein level was dramatically elevated in SW780 and BIU87 cells (Fig. 2b). To further determine the effect of radiation treatment on TUG1 and HMGB1 expression, SW780 and BIU87 cells were treated with 2 Gy of radiation. qRT-PCR analysis manifested that the TUG1 expression in SW780 and BIU87 cells (Fig. 2c and $\mathrm{d}$ ) was dramatically increased after radiation treatment in time- and dose-dependent manners. Western blot results indicated that the HMGB1 protein level was increased after radiation exposure in both SW780 and BIU87 cells (Fig. 2e). Taken together, all these results revealed that radiation treatment promotes the TUG1 and HMGB1 expression in bladder cancer cell lines.
TUG1 knockdown enhances radiosensitivity of bladder cancer cell lines

Considering that radiation treatment could enhance the TUG1 expression (Fig. 2c), we assumed that TUG1 may be related to the radiation resistance in bladder cancer. The colony survival assay is considered as a canonical standard to determine radiosensitivity [25]. Moreover, the anti-apoptosis activities of cancer cells are closely related to radioresistance. To confirm whether TUG1 could affect the radiosensitivity of bladder cancer cells, loss-offunction assay was performed by transfecting si-TUG1 (siRNAs specific to TUG1) into SW780 and BIU87 cells. Among the designed three siRNAs (si-TUG1\#1, siTUG1\#2 and si-TUG1\#3), si-TUG1\#3 was validated to possess the highest knockdown efficiency in SW780 and BIU87 cells (Fig. 3a) and was subsequently used for further experiments. SW780 and BIU87 were transfected with si-NC or si-TUG1 and incubated for $24 \mathrm{~h}$, then the cells were exposed to 2 Gy of irradiation. At indicated time points, cells were used for proliferation and apoptosis analysis. CCK-8 assay manifested that TUG1 knockdown markedly impaired the cell growth of SW780 and BIU87 cells compared with control group (Fig. 3b). Flow cytometry analysis revealed that the apoptosis rates of SW780 and BIU87 cells were prominently enhanced by TUG1 silencing (Fig. $3 \mathrm{c}$ and d).
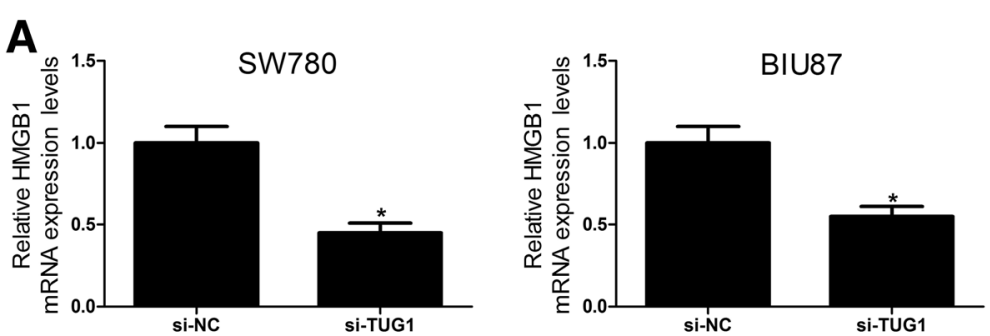

B
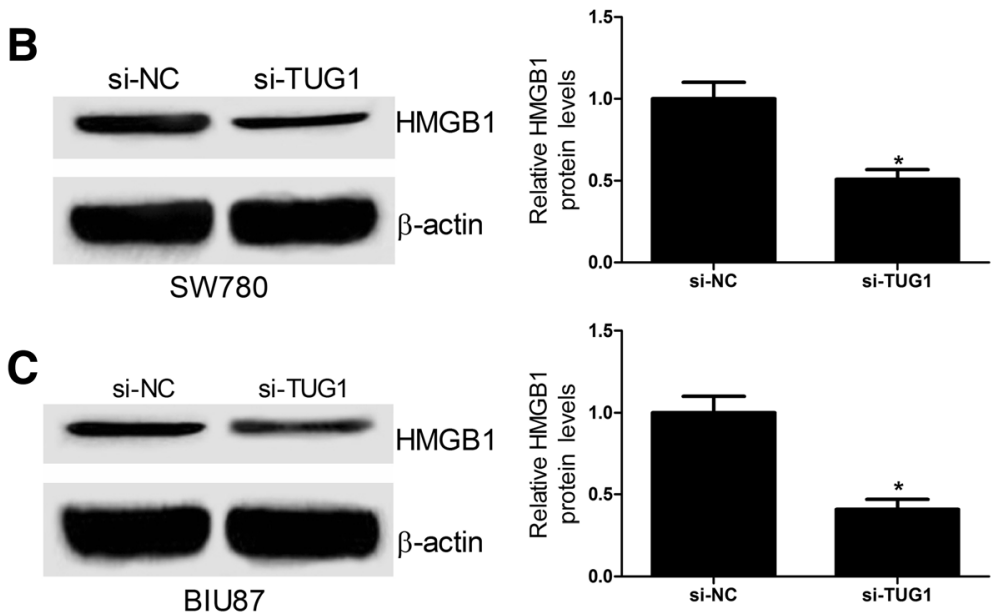

Fig. 4 TUG1 downregulation inhibits the HMGB1 expression in bladder cancer cell lines. a The expression of HMGB1 mRNA was detected by qRT-PCR analysis at $24 \mathrm{~h}$ after SW780 and BIU87 cells were transfected with si-NC or si-TUG1. b and c The protein level of HMGB1 was examined at $24 \mathrm{~h}$ after SW780 and BIU87 cells were transfected with si-NC or si-TUG1. ${ }^{*} P<0.05$ vs. si-NC 
Moreover, clonogenic assay indicated that TUG1 downregulation significantly lowered the survival fractions in SW780 and BIU87 cells (Fig. 3e) compared with controls. Collectively, these data demonstrated that TUG1 knockdown enhances radiosensitivity of bladder cancer cells SW780 and BIU87.

\section{TUG1 knockdown inhibits the HMGB1 expression in}

\section{bladder cancer cell lines}

Considering the positive correlation between TUG1 and HMGB2 expression in bladder cancer tissues and cells, as well as their consistent change under radiation treatment, we assumed that TUG1 may affect the radiosensitivity of bladder cancer cells via regulating the expression of HMGB2. To verify this hypothesis, the effect of TUG1 knockdown on HMGB1 expression in bladder cancer cells was first assessed., qRT-PCR and western blot showed that the mRNA (Fig. 4a) and protein (Fig. 4b and c) levels of HMGB1 were dramatically downregulated in si-TUG1transfected SW780 and BIU87 cells. All these results illustrated that silencing of TUG1 inhibited the HMGB1 expression in bladder cancer cell lines.

\section{Restoration of HMGB1 expression relieves the enhanced radiosensitivity of bladder cancer cells caused by TUG1} To further investigate whether TUG1 knockdown enhanced radiosensitivity of bladder cancer cells via inhibiting HMGB1, SW780 and BIU87 cells transfected with si-TUG1 or combination of si-TUG1 and pcDNAHMGB1 were treated with 2 Gy radiation. Restored expression of HMGB1 by pcDNA-HMGB1 reversed the inhibited cell viability (Fig. 5a), increased apoptosis
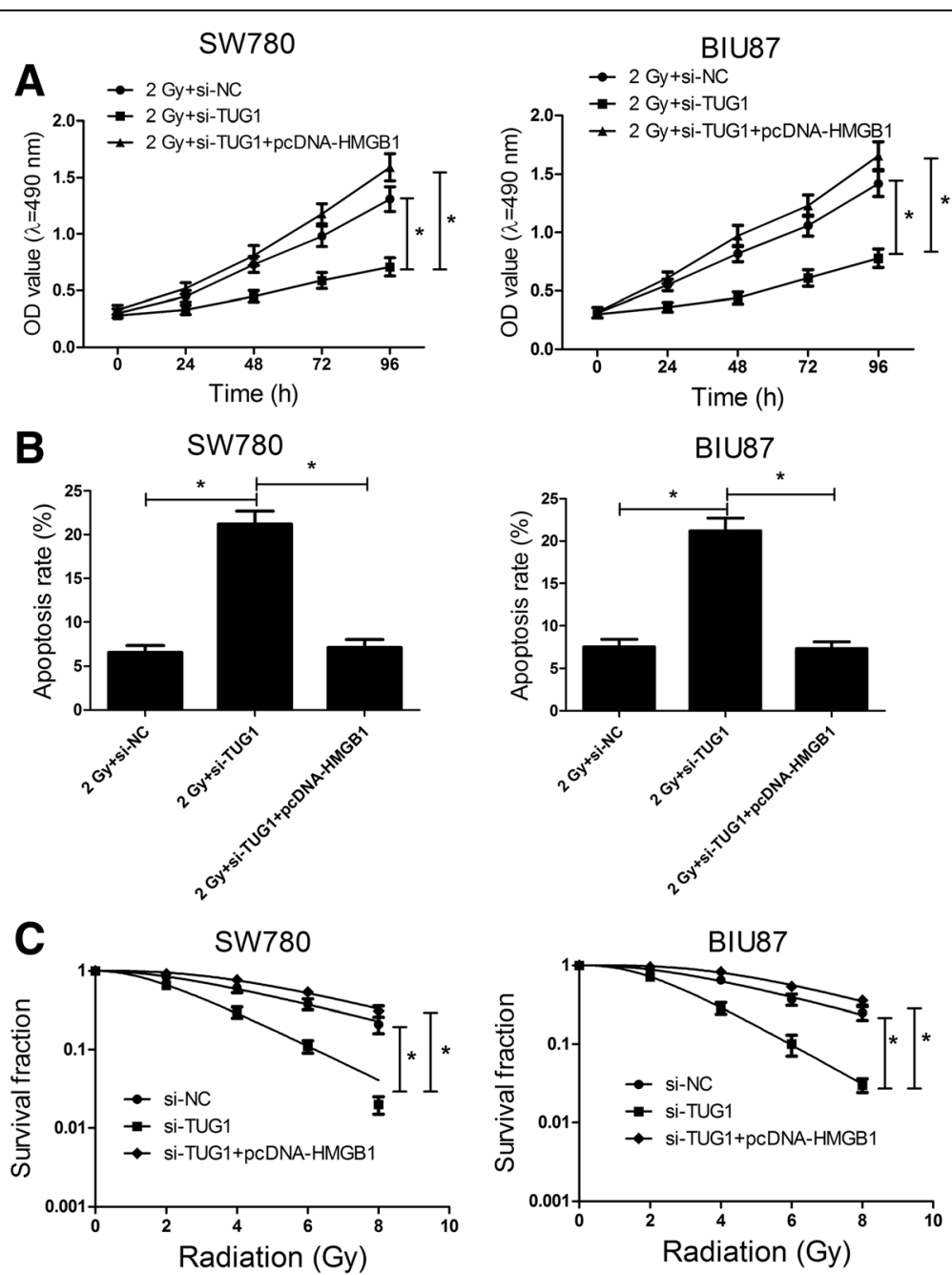

Fig. 5 Restoration of HMGB1 expression reversed the increased radiosensitivity of bladder cancer cells induced by TUG1 knockdown. SW780 and BIU87 cells were transfected with si-TUG1 or co-transfected with si-TUG1 and pcDNA-HMGB1. At $24 \mathrm{~h}$ post transfection, cells were exposed to Xray for radiation. a Cell proliferation was detected using CCK-8 Kit at the indicated times after 2 Gy of irradiation treatment. b Cell apoptosis was tested by flow cytometry at $24 \mathrm{~h}$ after radiotherapy. $\mathbf{c}$ The clonogenic survival curve was established on day 14 after transfected cells received indicated doses of radiation. ${ }^{*} P<0.05$ vs. Si-NC 
(Fig. 5b) and reduced survival fractions (Fig. 5c) induced by the depletion of TUG1 in SW780 and BIU87 cells. To sum up, these results revealed that knockdown of TUG1 enhances radiosensitivity of bladder cancer cells by downregulating HMGB1 expression.

\section{TUG1 knockdown sensitized bladder cancer cells to irradiation in vivo}

To determine the effect of TUG1 knockdown on the radiosensitivity of the bladder cancer mouse model, nontransfected or transfected SW780 cells were injected subcutaneously into nude mice. When the xenografts reached a certain volume, the mice received a 2 Gy irradiation treatment for successive 5 days. The results demonstrated that radiation treatment significantly inhibited the tumor growth, including the average volume (Fig. 6a) and weight (Fig. 6b), and this effect was more evident when combined with TUG1 knockdown. Together, TUG1 depletion improved the radiosensitivity of bladder cancer cells in vivo.

\section{Discussion}

In the present study, we explored function and underlying mechanisms of lncRNA TUG1 in the radiosensitivity of bladder cancer. Our study declared that TUG1 expression was markedly upregulated in bladder cancer tissues and cell lines. Moreover, TUG1 knockdown enhanced the radiosensitivity of bladder cancer cell lines, evidenced by the reduced cell viability, the increased cell apoptosis and the inhibited colony survival fractions. Furthermore, our findings suggest that TUG1 knockdown enhances radiosensitivity of bladder cancer cells in vivo and in vitro by suppressing the expression of HMGB1.
LncRNA TUG1 was overexpressed in several kinds of cancer tissues such as esophageal carcinoma [20], osteosarcoma [22] and hepatocellular carcinoma [26]. However, TUG1 was downregulated in non-small cell lung cancer and glioma [21, 27]. All these studies revealed that TUG1 could function as an oncogene or tumor suppressor in different cancers. In the present study, we found that TUG1 expression was increased in bladder cancer tissues and cell lines, which was consistent with the data of previous studies [22-24]. Moreover, radiation increased TUG1 expression, which implied that TUG1 may be associated with the radioresistance in bladder cancer. TUG1 knockdown inhibited cell proliferation and colony survival fractions, as well as induced cell apoptosis, which was supported by a previous finding that TUG1 inhibition upregulated the expression of the apoptosis-inducing factors such as AIF, AIP1, NIP3, and NIPOR, and suppressed cell growth and promoted apoptosis [28]. Additionally, radiation significantly blocked the tumor growth of mouse model, and the inhibitory effect was enhanced when combined with TUG1 knockdown. All these findings demonstrate that TUG1 knockdown contributes to the increase of radiosensitivity of bladder cancer cells in vivo and in vitro. It was reported that TUG1 promoted radioresistance of bladder cancer [24], which agrees with our findings.

HMGB1 was found to be overexpressed and could regulate tumor growth, metastasis and survival in cancers, including bladder cancer [29]. HMGB1 is also related to the chemoresistance and radioresistance in various cancers [30-32]. For instance, HMGB1 could mediate the chemotherapy resistance in breast cancer [11]. HMGB1 knockdown could promote the radiosensitivity of breast cancer cells through breaking
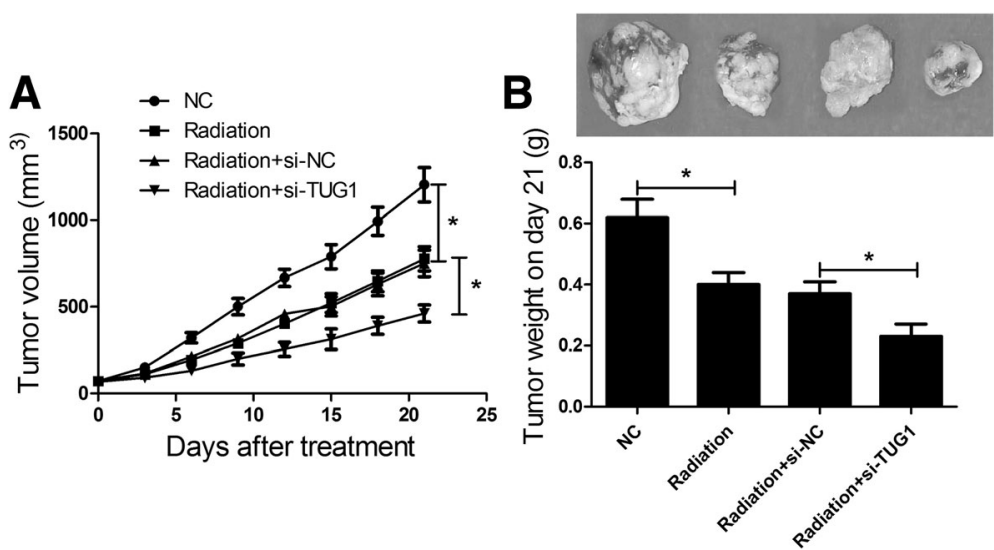

Fig. 6 TUG1 knockdown enhances radiosensitivity of bladder cancer cells in vivo. SW780 cells were subcutaneously injected into the back of male athymic nude mice ( $n=6$ per group). All mice were given radiation ( $2 \mathrm{~Gy}$ ) for 5 consecutive days when the tumors reached an average volume of about $100 \mathrm{~mm}^{3}$. a Tumor volume was measured every 3 days after the first radiotherapy. b Mice were euthanatized to resect and weigh tumors after 3 weeks. ${ }^{*} P<0.05$ vs. NC or si-NC 
telomere homeostasis and inhibiting the repair of DNA damage [12]. Moreover, HMGB1 knockdown markedly sensitize the bladder cancer cells to radiotherapy [33]. However, how it is regulated in radiosensitivity of bladder cancer is not quite clear. This study presented a positive correlation between TUG1 and HMGB1 expression, and their changes were consistant in response to irradiation treatment. Therefore, we explored whether TUG1 regulated the radiosensitivity of bladder cancer through modulating HMGB1 expression. In consistent with our surmise, our findings reveal that IncRNA TUG1 knockdown enhances radiosensitivity of bladder cancer through suppressing HMGB1 expression. A previous study found that TUG1 promoted cancer cell invasion and radioresistance through inducing epithelialto-mesenchymal transition (EMT) in bladder cancer [24]. In addition, a previous document revealed that elevated TUG1 expression was closely correlated with chemotherapy resistance of esophageal squamous cell carcinoma [28]. Therefore, TUG1 may be involved in the chemotherapy and radiotherapy resistance in cancers, providing a theoretical foundation for the clinical application of TUG1 in patients with radio- and chemo-resistant cancer.

\section{Conclusions}

In summary, TUG1 expression was upregulated in bladder cancer tissues and cell lines. Downregulation of TUG1 enhanced the radiosensitivity of bladder cancer cells via inhibiting HMGB1 expression. Our findings suggest that TUG1 could be a promising therapeutic target for bladder cancer and combination treatment of TUG1 knockdown and radiation may be a better strategy for the patients with radioresistant bladder cancer.

\section{Abbreviations}

CRC: Colorectal cancer; EMT: Epithelial-to-mesenchymal transition; HMGB1: High mobility group box 1 protein; LncRNAs: Long non-coding RNAs; MIBC: Muscle invasive bladder cancer; NMIBC: Non-muscle invasive bladder cancer; PDAC: Pancreatic ductal adenocarcinoma; qRTPCR: quantitative real-time PCR; TUG1: Taurine-upregulated gene 1; TUG1: Taurine-upregulated gene 1; WIF-1: Wnt inhibitory factor 1

\section{Acknowledgements}

None.

\section{Funding}

Not applicable.

\section{Availability of data and materials}

Please contact author for data requests.

\section{Authors' contributions}

$\mathrm{XH}$ designed and performed the experiment. $\mathrm{HZ}$ and $\mathrm{WL}$ analyzed the data and wrote the manuscript. HJ supervised the study and reviewed the manuscript. All authors read and approved the final manuscript.

\section{Competing interests}

The authors declare that they have no competing interests.
Ethics approval and consent to participate

Ethics approval and consent to participate were obtained from Huaihe hospital in Henan province.

\section{Publisher's Note}

Springer Nature remains neutral with regard to jurisdictional claims in published maps and institutional affiliations.

Received: 7 December 2016 Accepted: 26 March 2017

Published online: 04 April 2017

\section{References}

1. Chavan S, Bray F, Lortet-Tieulent J, Goodman M, Jemal A. International variations in bladder cancer incidence and mortality. Eur Urol. 2014;66:59-73.

2. Chang JS, Lara PN, Pan C-X. Progress in personalizing chemotherapy for bladder cancer. Adv Urol. 2012;2012:364919.

3. Celona B, Weiner A, Di Felice F, Mancuso FM, Cesarini E, Rossi RL, et al. Substantial histone reduction modulates genomewide nucleosomal occupancy and global transcriptional output. PLoS Biol. 2011;9:e1001086.

4. Reeves R. Nuclear functions of the HMG proteins. Biochim Biophys Acta. 1799;2010:3-14

5. Kang R, Livesey KM, Zeh I, Herbert J, Loze MT, Tang D. HMGB1: a novel Beclin 1-binding protein active in autophagy. Autophagy. 2010;6:1209-11.

6. Scaffidi P, Misteli T, Bianchi ME. Release of chromatin protein HMGB1 by necrotic cells triggers inflammation. Nature. 2002:418:191-5.

7. Mardente S, Mari E, Consorti F, Di Gioia C, Negri R, Etna M, et al. HMGB1 induces the overexpression of miR-222 and miR-221 and increases growth and motility in papillary thyroid cancer cells. Oncol Rep. 2012;28:2285-9.

8. Yan W, Chang Y, Liang X, Cardinal JS, Huang H, Thorne SH, et al. Highmobility group box 1 activates caspase-1 and promotes hepatocellular carcinoma invasiveness and metastases. Hepatology. 2012;55:1863-75.

9. $\quad$ Yang $L, Y u ~ Y$, Kang $R$, Yang $M$, Xie $M$, Wang Z, et al. Up-regulated autophagy by endogenous high mobility group box-1 promotes chemoresistance in leukemia cells. Leuk Lymphoma. 2012;53:315-22.

10. Yao X, Zhao G, Yang H, Hong X, Bie L, Liu G. Overexpression of highmobility group box 1 correlates with tumor progression and poor prognosis in human colorectal carcinoma. J Cancer Res Clin Oncol. 2010;136:677-84.

11. Amornsupak K, Insawang T, Thuwajit P, Pornchai O, Eccles SA, Thuwajit C. Cancer-associated fibroblasts induce high mobility group box 1 and contribute to resistance to doxorubicin in breast cancer cells. BMC Cancer. 2014;14:1.

12. Ke S, Zhou F, Yang H, Wei Y, Gong J, Mei Z, et al. Downregulation of high mobility group box 1 modulates telomere homeostasis and increases the radiosensitivity of human breast cancer cells. Int J Oncol. 2015;46:1051-8.

13. Zhu Y, Luo M, Brooks M, Clouthier SG, Wicha MS. Biological and clinical significance of cancer stem cell plasticity. Clin Transl Med. 2014;3:32.

14. Prensner JR, Chinnaiyan AM. The emergence of IncRNAs in cancer biology. Cancer Discov. 2011;1:391-407.

15. Qiu M-T, Hu J-W, Yin R, Xu L. Long noncoding RNA: an emerging paradigm of cancer research. Tumor Biol. 2013;34:613-20.

16. Jiang Y, Li Z, Zheng S, Chen H, Zhao X, Gao W, et al. The long non-coding RNA HOTAIR affects the radiosensitivity of pancreatic ductal adenocarcinoma by regulating the expression of Wnt inhibitory factor 1. Tumor Biol. 2016:37:3957-67.

17. Lu H, He Y, Lin L, Qi Z, Ma L, Li L, et al. Long non-coding RNA MALAT1 modulates radiosensitivity of HR-HPV+ cervical cancer via sponging miR145. Tumor Biol. 2016;37:1683-91.

18. Yang X-D, Xu H-T, Xu X-H, Ru G, Liu W, Zhu J-J, et al. Knockdown of long non-coding RNA HOTAIR inhibits proliferation and invasiveness and improves radiosensitivity in colorectal cancer. Oncol Rep. 2016;35:479-87.

19. Xu Y, Wang J, Qiu M, Xu L, Li M, Jiang F, et al. Upregulation of the long noncoding RNA TUG1 promotes proliferation and migration of esophageal squamous cell carcinoma. Tumor Biol. 2015;36:1643-51.

20. Zhang $Q$, Geng P-L, Yin P, Wang X-L, Jia J-P, Yao J. Down-regulation of long non-coding RNA TUG1 inhibits osteosarcoma cell proliferation and promotes apoptosis. Asian Pac J Cancer Prev. 2013;14:2311-5.

21. Zhang E, Yin D, Sun M, Kong R, Liu X, You L, et al. P53-regulated long noncoding RNA TUG1 affects cell proliferation in human non-small cell lung cancer, partly through epigenetically regulating HOXB7 expression. Cell Death Dis. 2014;5:e1243. 
22. Han Y, Liu Y, Gui Y, Cai Z. Long intergenic non-coding RNA TUG1 is overexpressed in urothelial carcinoma of the bladder. J Surg Oncol. 2013; 107:555-9.

23. Hiev R, Kleinova R, Juracek J, Dolezel J, Ozanova Z, Fedorko M, et al. Overexpression of long non-coding RNA TUG1 predicts poor prognosis and promotes cancer cell proliferation and migration in high-grade muscleinvasive bladder cancer. Tumor Biol. 2016;37:13385-90.

24. Tan J, Qiu K, Li M, Liang Y. Double-negative feedback loop between long non-coding RNA TUG1 and miR-145 promotes epithelial to mesenchymal transition and radioresistance in human bladder cancer cells. FEBS Lett. 2015;589:3175-81.

25. Yaromina A, Krause M, Thames H, Rosner A, Krause M, Hessel F, et al. Pretreatment number of clonogenic cells and their radiosensitivity are major determinants of local tumour control after fractionated irradiation. Radiother Oncol. 2007;83:304-10

26. Huang M-D, Chen W-M, Qi F-Z, Sun M, Xu T-P, Ma P, et al. Long non-coding RNA TUG1 is up-regulated in hepatocellular carcinoma and promotes cell growth and apoptosis by epigenetically silencing of KLF2. Mol Cancer. 2015;14:1.

27. Li J, Zhang M, An G, Ma Q. LncRNA TUG1 acts as a tumor suppressor in human glioma by promoting cell apoptosis. Exp Biol Med (Maywood). 2016; 241:1644-9.

28. Jiang L, Wang W, Li G, Sun C, Ren Z, Sheng H, et al. High TUG1 expression is associated with chemotherapy resistance and poor prognosis in esophageal squamous cell carcinoma. Cancer Chemother Pharmacol. 2016; 78:333-9.

29. Yang GL, Zhang LH, Bo JJ, Huo XJ, Chen HG, Cao M, et al. Increased expression of HMGB1 is associated with poor prognosis in human bladder cancer. J Surg Oncol. 2012;106(1):57-61.

30. Zhang X, Qi L, Yang L. HMGB1 regulates autophagy and apoptosis to promote chemotherapy resistance in multiple myeloma. Cancer Res. 2014;74:680

31. Zheng H, Chen J-N, Yu X, Jiang P, Yuan L, Shen H-S, et al. HMGB1 Enhances Drug Resistance and Promotes in vivo tumor growth of lung cancer cells. DNA Cell Biol. 2016;35:622-7.

32. Luo J, Chen J, He L. mir-129-5p attenuates irradiation-induced autophagy and decreases radioresistance of breast cancer cells by targeting HMGB1. Med Sci Monit. 2015;21:4122-9.

33. Shrivastava S, Mansure JJ, Almajed W, Cury F, Ferbeyre G, Popovic M, et al. The Role of HMGB1 in radioresistance of Bladder Cancer. Mol Cancer Ther. 2016;15:471-9.

\section{Submit your next manuscript to BioMed Central and we will help you at every step:}

- We accept pre-submission inquiries

- Our selector tool helps you to find the most relevant journal

- We provide round the clock customer support

- Convenient online submission

- Thorough peer review

- Inclusion in PubMed and all major indexing services

- Maximum visibility for your research

Submit your manuscript at www.biomedcentral.com/submit 